\title{
The Effect of Upper Trapezius Latent Myofascial Trigger Points on Neck Pain and Disability
}

\author{
Magesh Anand Doraisamy (Corresponding author) \\ Physiotherapist/Assistant Manager \\ Masterskill Physiotherapy and Rehabilitation Center (Masterskill University College of Health Sciences) \\ G8 Jalan kemacahaya, 11 Taman kemacahaya \\ Batu 9 Cheras, Selangor, 43200, Malaysia \\ Tel: 60-149-244-935 E-mail: dmagesh@masterskill.edu.my, mageshanand@gmail.com
}

Anshul

Physiotherapist, Malaysia

Received: January 26, 2011 Accepted: February 10, 2011 doi:10.5539/gjhs.v3n2p134

\begin{abstract}
Purpose: To compare neck pain and disability between people with and without latent myofascial trigger points (MTrP) in Upper Trapezius. Methods: The participants of the study were asked to fill the Northwick Park Neck Pain Questionnaire (NPNQ) following which bilateral upper trapezius muscles were assessed for the presence of latent MTrP. The participants were placed into two groups; an experimental group who had latent MTrP in upper trapezius and a control group who did not have trigger points. Twenty seven people (22 women and 5 men, age range 18 to 22 years, mean age 19.62 +/- 1.33 years) were in the experimental group and Fifteen people (10 women and 5 men, age range 18 to 23 years, mean age 18.86 +/- 1.55 years) were in the control group. Analysis: The scores from the NPNQ was compared between the two groups using unpaired ' $t$ ' test. Results: The results showed that the neck pain and disability is significantly higher in people with latent MTrP than the people without Latent MTrP. Conclusion: The latent MTrP despite of being quiescent might have a significant role to play in influencing neck pain and disability.
\end{abstract}

Keywords: Latent myofascial trigger point, Upper trapeziums

\section{Introduction}

Myofascial pain syndrome is a complex pain disorder characterized by a steady dull ache referring to a specific reference zone from a myofascial trigger point within a palpable band of muscle (Simons et al, 1999). A myofascial trigger point (MTrP) is a hyperirritable spot, located within a taut band of a skeletal muscle that is painful on compression or stretch and that can give rise to a typical referred pain pattern as well as autonomic phenomena (Simons et al., 1999; Elizabeth et al., 2007). Myofascial trigger points are further grossly subdivided as latent MTrP and active MTrP.

A latent myofascial trigger point is known as "A myofascial trigger point that is clinically quiescent with respect to spontaneous pain; it is painful only when palpated. A latent trigger point may have all the other clinical characteristics of an active trigger point and always has a taut band that increases muscle tension and restricts range of motion" (Simons et al., 1999). An active myofascial trigger point is known as "A myofascial trigger point that causes a clinical pain complaint. It is always tender, prevents full lengthening of the muscle, weakens the muscle, refers a patient-recognized pain on direct compression, mediates a local twitch response of the muscle fibers when adequately stimulated and when compressed within the patients' pain tolerance produces referred motor phenomena and often autonomic phenomena generally in its pain reference zone and causes tenderness in the pain reference zone” (Simons et al., 1999).

It is observed in few studies; some dating back to the early $20^{\text {th }}$ century that the prevalence of latent MTrP are quite common. Two hundred asymptomatic young adults were assessed and found to have focal tenderness representing latent trigger points in the shoulder girdle muscles of $54 \%$ of the female and $45 \%$ of the male 
subjects. Referred pain was demonstrated in $25 \%$ of these subjects with latent trigger points (Sola et al., 1955). The lumbo-gluteal muscles in 100 asymptomatic control subjects were examined and found to have latent trigger points in quadratus lumborum (45\% of the subjects), gluteus medius (41\%), iliopsoas (23\%), gluteus minimus (11\%) and piriformis (5\%) (Frohlich et al., 1995).The presence of latent myofascial trigger points in the scapular positioning muscles were examined in 154 healthy adults and nearly $90 \%$ of the population were found to have at least one trigger point in the muscles assessed (Lucas et al., 2008).

Latent MTrP are clinically significant for the fact that they remain quiescent; hence a person will not be even aware of the presence of the trigger point which might render them weaker and more susceptible to fatigue. Though latent MTrP are found to be quite prevalent there is a lack of literature to associate the presence of latent MTrP with neck pain and disability assessed with a standardized questionnaire. The effect of latent MTrP on neck pain and disability has been largely unexplored. Hence this study was done with the aim to observe the presence of latent MTrP on people with neck pain and compare the neck pain and associated disability between people with and without latent MTrP using the NPNQ questionnaire.

\section{Methods}

\subsection{Subjects}

An advertisement about the study was put up to the Faculty of Therapeutic Sciences, Masterskill University College of Health Sciences, Selangor, Malaysia. The people willing to participate in the study were asked to report to the principal investigator. The study was done at Masterskill Physiotherapy and Rehabilitation Center, Selangor, Malaysia. Forty two people with mechanical neck pain for at least the past 3 months participated in this study. For the purpose of this study, mechanical neck pain was defined as generalized neck pain with mechanical characteristics including: symptoms provoked by prolonged neck postures or by movement. The exclusion criteria was set up as, previous history of whiplash injury, history of cervical spine surgery, diagnosis of cervical radiculopathy or myelopathy, signs and symptoms suggestive of active myofascial trigger points, therapeutic intervention for myofascial pain within the past month and diagnosis of fibromyalgia syndrome according to the criteria set by American College of Rheumatology. The exclusion criteria were determined by interview and physical examination by a physiotherapist. The current health status was ascertained to be normal and without any concomitant disease. The study was approved by the institutional research board and a written informed consent was signed by all the participants prior to taking part in the study.

\subsection{Procedure}

The participants of the study were given the NPNQ questionnaire printed in English. They were advised to seek help from the principal investigator if they were not clear with any of the components of the questionnaire. The NPNQ questionnaire consists of nine five-part sections with a minimum score of zero and a maximum score of 4 . The questions are designed in such a way that it covers the activities which are likely to be affected by neck pain. All the participants were instructed to tick only the one box that closely describes their current situation. After filling up the questionnaire the subjects were assessed for the presence of latent MTrP in the upper trapezius.

The subjects were assessed for the presence of latent MTrP in the upper trapezius by a physical therapist with more than three years experience in the assessment and management of myofascial pain syndrome. To assess the trigger point the subject was positioned in high sitting with the arms supported on their lap. The therapist palpated the entire stretch of the muscle from the origin at the cervical spine to the insertion of the muscle fibers at the medial border of the lateral acromion process. The minimum essential criteria recommended in the literature (Simons et al., 1999) were followed for assessment and diagnosis of a latent trigger point. The physical signs considered included: presence of a palpable taut band or a nodule in the skeletal muscle, an exquisite tender spot in the taut band/nodule, a positive jump sign and referred pain to an area away from the site of trigger point. The people with latent MTrP were placed in the experimental group and the people without latent $\mathrm{MTrP}$ were placed in the control group.

\subsection{Data Analysis}

All the components in the NPNQ questionnaire are assessed over five parts from zero to four, the zero being the lowest and four the highest possible score for a given component. The mean and S.D. of each of the components was calculated (please refer figure 1). The scores of each of the parameters of the 9 item NPNQ questionnaire was compared between the experimental and control group. The data analysis was done with the SPSS statistical package (11.5 versions). Unpaired' $t$ ' test with a $95 \%$ confidence interval was performed. A p-value of less than 0.05 was considered to be statistically significant. 


\section{Results}

A total of 42 people participated in this study with 27 people in the experimental group (22 women and 5 men, age range 18 to 22 years, mean age 19.62 +/- 1.33 years) and 15 people (10 women and 5 men, age range 18 to 23 years, mean age $18.86+/-1.55$ years) in the control group. The results from the statistical analysis showed that except for the component on pins and needles sensation or numbness in the arm at night, the difference between the scores obtained from all the other parameters were statistically significant between the two groups. (Please refer table 1).

In the component "neck pain intensity" the subjective status of pain of the individual during the time of assessment was asked. The mean of the control group was 0.13 and the experimental group was 0.89 . The difference of neck pain intensity scores between the two groups was statistically significant. It can be inferred from the analysis that majority of the people in the control group did not have any pain at the time of assessment where as in the experimental group majority of the people had mild pain at the time of assessment. The component "neck pain and sleeping" reflects the sleep being disturbed by the neck pain. The mean score of people in the control group was 0.27 while the mean of the experimental group was 0.93 . The difference between the groups was statistically significant which showed that majority of the people in the experimental group have had more disturbances in sleep because of pain than when compared to the control group.

In the component that dealt with "pins and needles sensation and numbness during the night" the mean of the control group was 0.4 and the mean of the experimental group was 0.59 . The difference between the two groups was not statistically significant. Further it of concern to observe this type of pain in this group since it is commonly associated with nerve/nerve root injuries. In the component "duration of symptoms" the subjects were asked to reflect on the time period of discomfort in their neck and arm in a day. The mean of the control group was 0.53 and the mean of the experimental group was 1.18 . The difference was statistically significant which showed that majority of people in the control group felt their neck and arm normal all day while majority of the people in the experimental group had symptoms in their neck and arm lasting at less than one hour while some of them have had symptoms lasting $1-4$ hours of their waking time.

In the component "carrying" the ability of the people to carry heavy weights was asked. The mean of people in control group was 0.53 where as the mean of the experimental group was 0.96 . The difference was statistically significant which showed that majority of the people in control group could carry heavy objects without extra pain where as the people in experimental group can carry heavy objects but they give them extra pain.

In the component "reading and watching TV" the ability of the people to do this activity over a prolonged period of time was asked. The control group had a mean score of 1 where as the experimental group had a mean score of 1.66. The difference was statistically significant which showed that majority of people in the control group can do this activity for as long as they wished provided they are in a suitable position where as the people in experimental group can do this activity as long as they wished but it caused them extra pain.

In the component "working/housework" the ability of the people to do work without pain was asked. The people in the control group had a mean of 0.26 where as the mean of the experimental group was 1.07. The difference between the groups was statistically significant which showed that majority of the people in the control group can do their usual work without any extra pain while in the experimental group majority of the people can do their usual work but it gave them extra pain while some of them could not do their usual work for more than half the usual time due to pain.

In the component "social activities" the ability of the people to go about with their social life is asked. The control group had a mean of 0.27 which showed that majority of the people felt their social life is normal. The mean of the experimental group was 0.59 which showed that some of the people with latent MTrP felt that their social life is normal but it increases the degree of pain. In the component "driving" the ability of the people to drive without discomfort was asked. The control group had a score of 0.2 where as the mean of the experimental group was 0.85 . The difference was statistically significant which showed that majority of the people in the control group were able to drive whenever necessary without any discomfort where as in the experimental group majority of the people were able to drive whenever necessary but with discomfort.

\section{Discussion}

In the management of neck pain, the latent MTrP is often unaddressed, partly due to the fact that the latent $\mathrm{MTrP}$ are often quiescent. In the clinical community a latent MTrP is only considered to be a precursor for an active MTrP and hence are often left untreated. In this study it is observed that among the people with neck pain, those with latent MTrP have more neck pain and disability than those who did not have latent MTrP. This finding 
could imply that the increased pain and disability could be because of the latent MTrP. The effect of latent MTrP on neck pain and disability is revealed by the statistically significant differences in the scores obtained on the NPNQ questionnaire.

The effect of latent MTrP in upper trapezius on neck pain and associated disability can be partly explained by its effect on a skeletal muscle. In a biochemical point of view, elevated levels of sensitizing substances were found in the muscle tissues harboring the trigger points when compared to healthy muscle tissue (Shah et al., 2005; Shah et al., 2008). The presence of nociceptive and non-nociceptive hypersensitivity was showed at the site of latent $\mathrm{MTrP}$ (Li et al., 2009). A decreased blood flow was observed at the site of latent $\mathrm{MTrP}$ suggesting that it could be due to the increased vasoconstriction activity at the trigger point site (Zhang et al., 2008). In a mechanical point of view, an alteration in the muscle activation pattern (MAP) was found in the muscle group with a latent MTrP which was corrected following treatment. The presence of latent MTrP was shown to result in the active insufficiency of the sarcomeres under contracture (knot) and the lengthened sarcomeres outside the contraction knot which can affect the contractile capacity of the muscle (Lucas et al., 2004). These findings suggest that the latent MTrP negatively influences the skeletal muscle.

The upper trapezius plays an important role in the mobility and stability of the neck. During the various activities listed in the NPNQ questionnaire there should be a considerable muscle work from upper trapezius. The symptoms seen in people with latent MTrP could be explained by the energy crises theory (Simons et al., 1999). According to this theory, a sustained contractile activity of the sarcomeres (as seen in latent trigger points) would markedly increase the metabolic demands and would squeeze shut the rich network of capillaries that supply the nutritional and oxygen needs of that region. This concept has been substantiated by the finding that there is decreased blood flow in the muscle at the site of latent trigger point (Zhang et al., 2008).

The combination of increased metabolic demand and impaired metabolic supply could produce a severe but local energy crisis. The local hypoxia and tissue energy crises could stimulate production of vasoreactive substances that could sensitize local nociceptors and thus cause pain. Further, it is given in the literature that the presence of myofascial trigger points can lead to accelerated fatigability of the muscle (Hagberg and Kvarnstrom., 1984). In the presence of latent MTrP, the muscle could undergo an early fatigue and probably put excessive stress on the other stabilizing structures such as ligaments and capsules around the cervical joint causing pain.

In the component that described the pins and needles sensation during the night, the differences between the experimental and control group was not statistically significant but the presence of pins and needles sensation was observed in both groups. Pins and needles sensation is often associated with disorders relating to the nerves and nerve roots. Latent MTrP are not directly associated with causing compression of nerves or nerve roots causing pain similar to pins and needles sensation. The referred pain from the MTrP may not resemble the pins and needles sensation either. The people who were normal also complained of pins and needles sensation at night. It is of concern because the scores from both the groups should have ideally reflected that they do not have any pins and needles sensations.

However, it was observed from the questionnaire that all the participants in both groups had only marked the score of zero (no pins and needles sensation) or one (occasional pins and needles sensation at night).This component assesses the pins and needles sensation while lying down on the bed. The probable reason for the presence of pins and needles sensation could be because of transient endoneural anoxia which refers to decreased blood supply to the nerves owing to prolonged compression of the limbs resulting in ischemia and when the compression is removed the person would feel pins and needles sensation all over the limb for a short period of time. The arm could be compressed by the person's own head as in resting the head over the arms or in other positions where arm is caught up and compressed for a prolonged period of time. This could probably explain the reason for people having pins and needles sensation at night where either group should not have had this sensation at all owing to being normal and having a pathology that does not affect the nerves or nerve roots.

\section{Conclusion}

The majority of studies on myofascial trigger points are focused on pain related complaints which are more applicable to active MTrP. In this study the effect of latent trigger points on neck pain and disability was observed and a statistically significant difference was found. From a clinical standpoint, the result from this study supports the importance of assessing the upper trapezius for latent trigger points along with the other causative factors during the management of neck pain clients. 


\section{References}

Elizabeth, D. L., William, L., \& Howard, S. (2007). Myofascial trigger points. Med Clin North Am, 9, $229-239$.

Fröhlich, D. \& Fröhlich, R. (1995). Das Piriformis syndrom: eine häufige differential diagnose des lumboglutäalen schmerzes. Manuelle Medizin, 33, 7-10.

Hagberg, H. \& Kvarnstrom, S. (1984). Muscular endurance and electromyophic fatigue in myofascial shoulder pain. Arch Phys Med Rehabil, 65, 522-525.

Li, L. T., Ge, H. Y., Yue, S. W., \& Arendt-Nielsen, L. (2009). Nociceptive and non-nociceptive hypersensitivity at latent myofascial trigger points. Clin J Pain, 25, 132-137. http://dx.doi.org/10.1097/AJP.0b013e3181878f87

Lucas, K. R., Barbara, I., \& Peter, A. R. (2004). Latent myofascial trigger points: their effects on muscle activation and movement efficiency. J Bodyw Mov Ther, 8, 160-6. http://dx.doi.org/10.1016/j.jbmt.2003.12.002

Lucas, K. R., Peter, A. R., \& Barbara, I. P. (2008). How common are latent myofascial trigger points in the scapular positioning muscles. J Musculoskeletal Pain, 16, 279-286. http://dx.doi.org/10.1080/10582450802479800

Shah, J. P., Elizabeth, A., \& Gilliams, B. A. (2008). Uncovering the biochemical milieu of myofascial trigger points using in vivo microdialysis: an application of muscle pain concepts to myofascial pain syndrome. $J$ Bodyw Mov Ther, 12, 371-384.

Shah, J. P., Terry, M., Phillips, Jerome, V., \& Danoff Gerber, L. H. (2005). An in vivo micro analytical technique for measuring the local biochemical milieu of human skeletal muscle. J Appl Physiol, 99, 1977-1984. http://dx.doi.org/10.1152/japplphysiol.00419.2005

Simons, D. G., Travel, J., \& Simons, L. S. (1999). Myofascial pain and dysfunction: The trigger point manual-Volume 1, Upper half of body. 2nd ed. Baltimore: Williams and Wilkins.

Sola, A. B., Rodenberger, M. L., \& Gettys, B. B. (1955). Incidence of hypersensitive areas in posterior shoulder muscles. Am J Phys Med, 34, 585-590.

Zhang, Y., Ge, H. Y., Boudreau, S., Yue, S. W., \& Arendt-Nielsen, L. (2008). Induction of muscle cramps by ociceptive stimulation of latent myofascial trigger points. Exp Brain Res, 187, 623-629. http://dx.doi.org/10.1007/s00221-008-1331-y

Table 1. The table shows the mean and S.D. of the parameters observed in the NPNQ questionnaire and also the value obtained in the student ' $\mathrm{t}$ ' test

\begin{tabular}{|l|l|l|l|l|}
\hline S.no & Component & $\begin{array}{l}\text { Mean and S.D. of } \\
\text { control group }\end{array}$ & $\begin{array}{l}\text { Mean and S.D. of } \\
\text { Experimental group }\end{array}$ & $\begin{array}{l}\text { Student 't'test } \\
\text {-value (0.05) }\end{array}$ \\
\hline 1 & Neck pain intensity & $0.13+/-0.35$ & $0.88+/-0.89$ & 0.000 \\
\hline 2 & Neck pain and sleeping & $0.26+/-0.59$ & $0.92+/-0.61$ & 0.000 \\
\hline 3 & $\begin{array}{l}\text { Pins and needles or numbness in } \\
\text { the arms at night }\end{array}$ & $0.4+/-0.63$ & $0.59+/-0.63$ & 0.176 \\
\hline 4 & Duration of symptoms & $0.53+/-0.51$ & $1.18+/-0.96$ & 0.003 \\
\hline 5 & Carrying & $0.53+/-0.51$ & $0.96+/-0.70$ & 0.015 \\
\hline 6 & Reading and Watching TV & $1+/-0.65$ & $1.66+/-0.83$ & 0.003 \\
\hline 7 & Working/Housework & $0.26+/-0.45$ & $1.07+/-0.78$ & 0.000 \\
\hline 8 & Social activities & $0.26+/-0.45$ & $0.59+/-0.74$ & 0.043 \\
\hline 9 & Driving & $0.27+/-0.46$ & $0.88+/-0.6$ & 0.001 \\
\hline 10 & $\begin{array}{l}\text { Neck pain questionnaire } \\
\text { percentage }\end{array}$ & $10.40+/-6.72$ & $24.32+/-10.04$ & 0.000 \\
\hline
\end{tabular}




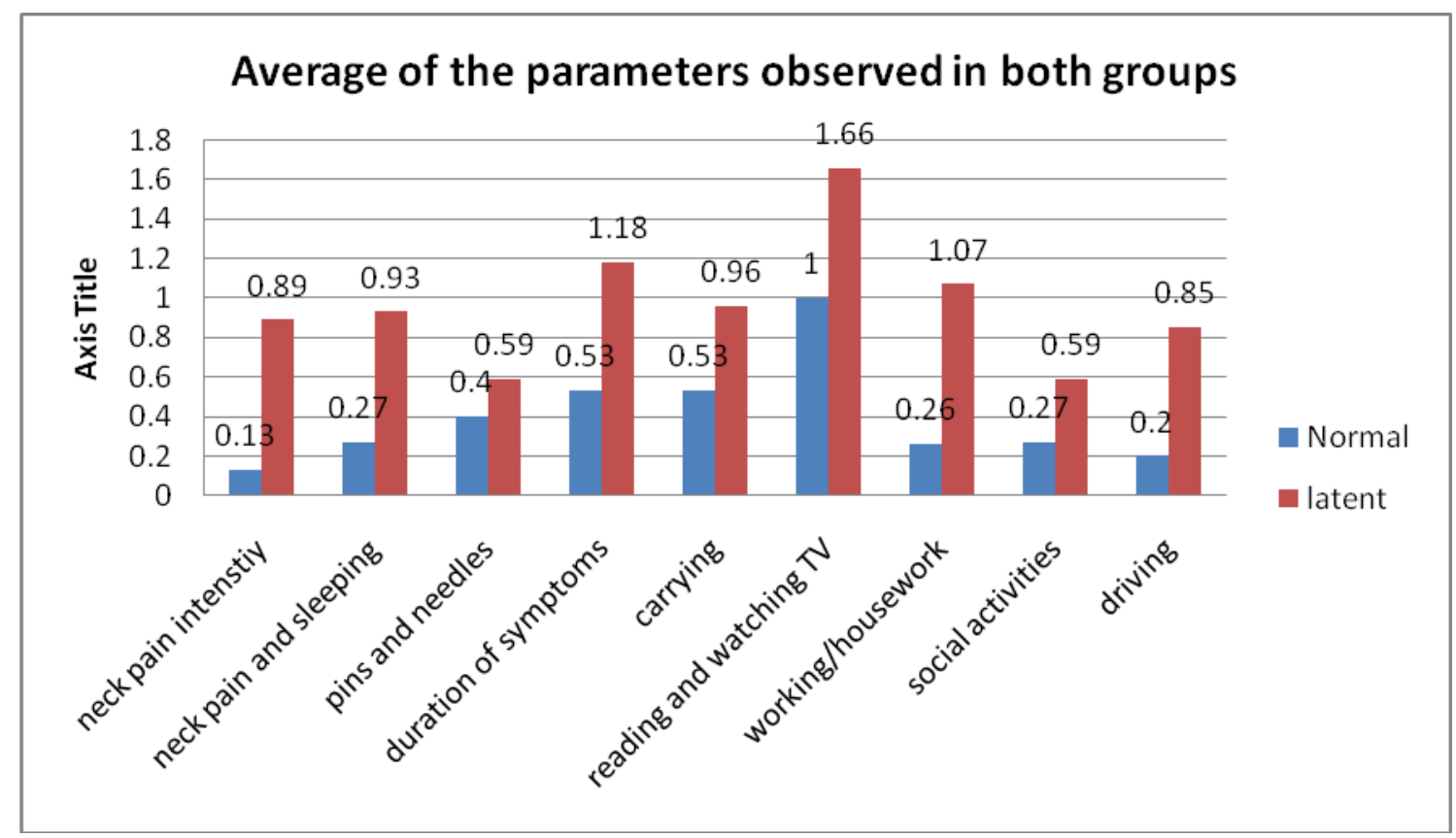

Figure 1. shows the mean value of the parameters observed in the NPNQ questionnaire 